\title{
Análisis del pensamiento creativo y niveles de activación del alumno tras una experiencia de gamificación
}

\author{
M.a Elena Parra-González \\ Adrián Segura-Robles \\ Universidad de Granada. España. \\ elenaparra@ugr.es \\ adrianseg@ugr.es

\section{Carmen Romero-García} \\ Universidad Internacional de la Rioja. España. \\ mariadelcarmen.romero@unir.net
}

Recibido: 3/2/2019

Aceptado: 16/1/2020

Publicado: 20/7/2020

\section{Resumen}

Los docentes se enfrentan a retos que necesitan de constante actualización, formación e información, y las tecnologías de la información y la comunicación (TIC) son elementos clave en ese asunto. En los entornos académicos virtuales es muy importante el conocimiento y el buen manejo de las TIC para que los procesos de enseñanza-aprendizaje sean eficaces. Una de las metodologías emergentes actuales es el uso de la gamificación en educación, que fomenta la motivación de los estudiantes cuando se enfrentan a problemas complejos contextualizados en la realidad profesional, a la vez que promueve una formación en competencias necesarias para su incorporación al mundo laboral. El objetivo de este estudio es medir qué factores han influido en el desarrollo de una experiencia gamificada. Para ello se ha hecho una investigación de corte cuantitativo, con un análisis descriptivo ex post facto a una población de 238 participantes distribuidos en 50 docentes y 188 estudiantes de distintas titulaciones de grado de universidades andaluzas. Entre los principales resultados se destacan los altos niveles de activación y de pensamiento creativo. La conclusión es que tanto estudiantes como docentes presentan grados elevados de motivación, de activación y de creatividad tras experiencias gamificadas.

Palabras clave: aprendizaje activo; innovación; metodologías activas; motivación, gamificación; educación superior

Resum. Anàlisi del pensament creatiu i nivells d'activació de l'alumne després d'una experiència de ludificació

Els docents s'enfronten a reptes que necessiten actualització, formació i informació constants, i les tecnologies de la informació i la comunicació (TIC) són elements clau en aquest assumpte. En els entorns virtuals d'aprenentatge és molt important conèixer i utilitzar les TIC correctament perquè els processos d'ensenyament-aprenentatge siguin eficaços. Una de les metodologies emergents actuals és l'ús de la ludificació en educació, que fomenta la motivació dels estudiants quan s'enfronten a problemes complexos contextualitzats en la realitat 
professional, alhora que promou una formació en competències necessàries per incorporar-se al món laboral. L'objectiu d'aquest estudi és mesurar quins factors han influït en el desenvolupament d'una experiència ludificada. Per aquest motiu s'ha fet una recerca de tall quantitatiu, amb una anàlisi descriptiva ex post facto a una població de 238 participants distribuïts en 50 docents i 188 estudiants de diferents titulacions de grau d'universitats andaluses. Entre els principals resultats se'n destaca els alts nivells d'activació i de pensament creatiu. Es por concloure que tant alumnes com docents presenten uns graus elevats de motivació, d'activació i de creativitat després d'experiències ludificades.

Paraules clau: aprenentatge actiu; innovació; metodologies actives; motivació; ludificació; educació superior

\title{
Abstract. Analysis of creative thinking and levels of student activation after a gamification experience
}

Teachers face many challenges that require constant updating, training and information and where information and communications technology (ICT) plays a key role. In virtual learning environments, knowledge and the appropriate use of ICT are very important to ensure effective teaching-learning processes. One of the emerging methodologies in this regard is the use of gamification in education. Gamification motivates students' when they are faced with complex problems contextualized in a professional setting and provides them training in the skills required for entry into the world of work. The objective of this article is to measure factors that influence a gamified experience. To this end, a quantitative study with an ex post-facto descriptive analysis was carried out. The study population comprised 238 participants: 50 teachers and 188 students from different degree programs at universities in Andalusia, Spain. The results revealed high levels of activation and creative thinking, indicating that both students and teachers achieve high levels of motivation, activation and creativity after gamified experiences.

Keywords: active learning; innovation; active methodologies; motivation; gamification; higher education

\author{
Sumario \\ 1. Introducción 5. Conclusiones \\ 2. Método 6. Futuras líneas de investigación \\ 3. Resultados Referencias bibliográficas
}

4. Discusión

\section{Introducción}

Hoy día la docencia se enfrenta a retos que requieren de constante actualización, formación e información, y para ello es necesario el uso efectivo de las tecnologías de la información y la comunicación (TIC) en los procesos de enseñanza-aprendizaje. Para que este uso sea eficaz y los alumnos puedan verse beneficiados, se necesita de la formación y del esfuerzo del profesorado (Martínez, Pérez y Martínez, 2016; Rubilar, Alveal y Fuentes, 2017). 
No son pocas las investigaciones (Davidson y Desjardins, 2011; Martins dos Santos y Mourão, 2017; Mirate y García, 2014) que aseguran que la integración de las TIC en educación conlleva desafíos para todos los participantes en ella, tanto para docentes como para alumnos y familias, y que la utilización de las TIC en el aula está vinculada a las más exitosas prácticas docentes (Ricoy y Couto, 2014). Por lo tanto, el uso de las TIC está asociado a la formación didáctica del docente en cuanto a metodologías a utilizar en el aula (Muñoz-Carril, Fuentes y González-Sanmamed, 2012).

Dentro de los entornos académicos virtuales es muy importante el conocimiento y el buen manejo y uso de las TIC para cualquier área, ya que ello va a ser de crucial importancia para que los procesos de enseñanza-aprendizaje resulten eficaces. Hay que aprovechar que los estudiantes de las nuevas generaciones ya poseen una relación bastante natural con las tecnologías y que incluso se aburren si no las usan a menudo (Lazzari, 2014). En la misma línea, autores como Rodríguez, Ramírez Leonardo y Fernández (2017) concluyen en su estudio que los alumnos se dispersan más cuando no se utiliza la tecnología en clase que cuando se recurre a ella.

En este sentido, una de las metodologías emergentes actuales es el uso de la gamificación en educación. La gamificación puede ser definida como el uso de estrategias, elementos y diseño de juegos en un contexto no lúdico (Deterding, Dixon, Khaled y Nacke, 2011; Werbach, 2014; Zainuddin, 2018). A esto Kapp (2012) añade que a través de esta metodología se desarrolla y aumenta la acción, el aprendizaje y la resolución de problemas. Otros autores defienden que, con el uso de la gamificación en clase, crece la motivación de los alumnos (Erenli, 2013; Lee y Hammer, 2011), con lo que se produce su aprendizaje. Wang (2015) también trabaja sobre cómo integrar el desarrollo del juego en educación, lo cual parece interesante dados los beneficios que se pueden obtener cuando unimos juegos y aprendizaje.

En su libro Homo ludens Huizinga (1955) analiza la importancia social y cultural de los juegos, cuyo ejercicio siempre ha acompañado a las personas. Según él, el juego no solo constituye una función humana tan esencial como la reflexión o el trabajo, sino que, además, la génesis y el desarrollo de la cultura poseen un carácter lúdico. Partiendo de esta idea, se ha utilizado el concepto de juego como una actividad libre de la vida cotidiana que interesa a los individuos. Con las tecnologías y con el uso de videojuegos ha aumentado la sensación de diversión y enganche de los usuarios (Brumels et al., 2008; Hassinger-Das et al., 2017). Hoy en día, con el uso de los teléfonos móviles y las redes sociales se extienden las noticias, las informaciones, las experiencias en videojuegos..., todo lo que nos podamos imaginar. La presencia de los juegos en nuestras vidas es algo real y frecuente, algo que no nos sorprende. Podemos plantear un futuro hipotético donde los videojuegos formarán parte de nuestras vidas, lo que constituye un proceso sin retorno. Quizás esta es una situación demasiado extrema, pero lo que sí es cierto es que está en nuestro entorno, que nuestros estudiantes se entretienen con videojuegos y que a las personas nos gusta jugar por naturaleza. Este elemento lo podríamos utilizar 
para que los aprendizajes fueran más divertidos, o al menos que se hicieran desde una actitud más desinhibida, como la de la predisposición a divertirse. Los estudiantes estarán atentos a los juegos y a sus dinámicas y aprenderán sin esfuerzo aparente, de manera más natural.

La gamificación viene de otras áreas y contextos, como el de las empresas, pero en los últimos años ha crecido su importancia y su utilidad en educación. $\mathrm{Al}$ introducir elementos y diseño de juegos en el trabajo diario con los estudiantes se busca aumentar su motivación (Shneiderman, 2004), pero para ello hay que planificar las actividades de forma coherente (Von Ahn y Dabbish, 2008).

Piñeiro-Otero y Costa-Sánchez (2015) defienden que con el uso de la gamificación en los procesos de enseñanza y aprendizaje se obtienen tantos beneficios que se adopta como metodología. Además, para obtener un determinado comportamiento de los estudiantes, estos deben estar motivados y al mismo tiempo capacitados para resolver los desafíos. De esta manera se está estableciendo una conexión entre el aprendizaje formal del entorno educativo con el mundo real en el que los estudiantes se desarrollan, con lo que se está fomentando su aprendizaje significativo.

Con este tipo de metodologías se pretende conseguir una participación en la que los alumnos cada vez sean más protagonistas de su propio aprendizaje, hecho muy importante en el proceso educativo (Méndez, 2008; Méndez y Trillo, 2008; Segura-Robles y Parra-González, 2019). Este tipo de metodologías centradas en el estudiante y de las cuales él es el protagonista activo pretenden también contribuir a desarrollar de manera productiva su componente motivacional, entendido como el proceso que le lleva hacia la meta de una actividad (Pintrich y Schunk, 2006). Así, en el proceso de enseñanza-aprendizaje el alumno tiene que ser el centro de atención.

Esta metodología es cada vez más popular y más utilizada en los entornos y en las instituciones educativas que buscan implementar en sus aulas metodologías más innovadoras o alternativas (Gómez-García, Planells de la Maza y Chicharro-Merayo, 2016; Parra-González y Segura-Robles, 2019). La gamificación se presenta como una estrategia idónea que permite la realización del aprendizaje por descubrimiento y la comprensión casi involuntaria de los contenidos en un ambiente creativo y agradable basado en la experiencia y en el juego. Se potencia en el alumnado la capacidad de pensar y razonar mediante la reflexión sobre sus propias decisiones, siendo el propio juego quien proporciona una respuesta rápida, coherente y concreta sobre las determinaciones tomadas. Se consigue además una profunda interiorización de lo aprendido y una formación de los estudiantes en competencias propias de su futuro profesional. Estos son los principales objetivos de cualquier docente (Teixes, 2015).

Como decíamos, no se puede establecer este tipo de metodología, ni ninguna otra, sin un conocimiento previo y una buena planificación de la misma. Así, Pérez-López y Rivera (2017) establecen que para el uso óptimo de esta metodología hay que tener en cuenta los elementos siguientes: crear un buen clima en el aula, implementar una metodología que esté centrada en la experiencia y el juego y utilizar la evaluación compartida. 
Como concluyen Aznar-Díaz, Raso-Sánchez, Hinojo-Lucena y RomeroDíaz de la Guardia (2016), los fututos docentes están convencidos de que la ludificación (o gamificación) será muy estimulante para los alumnos, que presentarán mayores niveles de entusiasmo. Además, el uso de videojuegos ejercerá una influencia que fomentará la adquisición de las competencias clave en su aprendizaje y desarrollo.

Este estudio va más allá de los previsibles resultados sobre el aumento de la motivación con el uso de la gamificación, puesto que se desprende de otros estudios que así lo demuestran. En realidad, pretende analizar cuáles son los niveles de pensamiento creativo y de activación del alumno tras una experiencia de gamificación educativa.

\section{Método}

Para la realización del presente estudio se ha realizado una investigación de corte cuantitativo y se ha llevado a cabo un análisis descriptivo ex post facto (Kothari, 2004), con una recogida muestral casual no probabilística.

\subsection{Participantes}

La muestra estuvo compuesta por un total de 238 participantes, entre los que había 50 docentes y 188 estudiantes de segundo y tercer cursos pertenecientes a las titulaciones de grado en Educación Primaria y en Educación Infantil de las universidades de Granada, Jaén y Sevilla. Durante la investigación nos aseguramos de que solo respondieran los participantes que habían intervenido en alguna experiencia gamificada.

\subsection{Instrumento}

Para la realización del estudio se diseñó un cuestionario dividido en dos partes. Por un lado, se recogieron una serie de variables sociodemográficas y, por otro, tres dimensiones extraídas de la escala GAMEX (Gameful Experience in Gamification), desarrollada y validada en inglés por Eppmann, Bekk y Klein (2018). El objetivo de este cuestionario era medir qué factores habían influido en el desarrollo de una experiencia gamificada. En nuestro caso, para llevar a cabo la presente investigación, seleccionamos tres de las dimensiones propuestas por los autores.

- Diversión: entendida como la capacidad de la actividad, el proyecto o la experiencia de gamificación para entretener a los participantes.

- Pensamiento creativo: entendido como la capacidad de la experiencia para ver y resolver problemas desde una perspectiva diferente, evitando soluciones ortodoxas.

- Actividad: entendida como la capacidad de la experiencia para despertar el interés o la concentración durante su desarrollo. 


\subsection{Procedimiento}

Para el proceso de recogida de datos el cuestionario se administró en línea a través de la aplicación Google Forms, plataforma gratuita y apropiada para trabajar a través de formularios (Rayhan et al., 2013). En cada universidad había un profesor encargado de difundir el enlace al cuestionario y asegurarse de que los informantes comprendían el objetivo de la actividad.

\subsection{Análisis de los datos}

En el análisis de los estadísticos descriptivos se incluye el análisis de las medias y las desviaciones estándar. La estadística descriptiva describe y resume las principales características observadas en un conjunto de datos a través de tablas, cuadros, gráficos y medidas de resumen, lo que permite al investigador comprender mejor el comportamiento de los datos. El análisis se basa en el conjunto de datos que se estudia (muestra), sin sacar conclusiones o inferencias de la población (Fávero y Belfiore, 2019).

En cuanto al cálculo de las diferencias y tras comprobar que la muestra utilizada no cumple los criterios estándar para dar un tratamiento a los datos desde la estadística paramétrica, en concreto el criterio de normalidad (Norman, 2010), se recurrió a los análisis propios de la estadística no paramétrica (Vickers, 2005). La estadística no paramétrica es un método en el que no se requiere que los datos se ajusten a una distribución normal, muy útil para las circunstancias en los que las muestras recogidas no cumplen uno o varios de los criterios de la estadística paramétrica (Corder y Foreman, 2009).

Además, en los casos en que las diferencias son significativas, se estiman los tamaños del efecto a través de la $d$ Cohen. Esto nos permite conocer la fuerza de las diferencias, y no solo describirlas en términos de magnitud (Cohen, 1987; Sullivan y Feinn, 2012).

\section{Resultados}

Con el objetivo de comprobar la validez del instrumento utilizado, se llevan a cabo distintas comprobaciones sobre la fiabilidad del mismo (tabla 1). Los valores obtenidos para el Alpha de Cronbach son $\alpha=0,89, \alpha=0,92$ y $\alpha=0,90$. Los que resultan superiores o cercanos a 0,90 son considerados índices aceptables de fiabilidad (Zumbo, Gadermann y Zeisser, 2007).

Por otro lado, la literatura científica ha criticado el uso exclusivo de este estadístico (Dunn, Baguley y Brunsden, 2013) como predictor de la garantía de una escala, por lo que se recomienda complementarlo con otros, como la fiabilidad compuesta (FC) o la varianza media extractada (VME). Los valores aceptables para estos estadísticos son aquellos que superan el nivel 0,7 para la FC y aquellos superiores a 0,50 para la VME (Hair y Gómez-Suárez, 2010). Como se muestra en la tabla 1 , los valores obtenidos para el factor activación $(\alpha=0,89 ; \mathrm{FC}=0,811 ; \mathrm{VME}=0,580)$, para el desarrollo del 
pensamiento creativo $(\alpha=0,92 ; \mathrm{FC}=0,913 ; \mathrm{VME}=0,640)$, para la diversión $(\alpha=0,90 ; \mathrm{FC}=0,899 ; \mathrm{VME}=0,630)$ y para el total de la escala utilizada $(\alpha=0,93 ; \mathrm{FC}=0,785 ; \mathrm{VME}=0,594)$ son propios de una escala con índices de fiabilidad aceptables.

Tabla 1. Análisis de fiabilidad de la escala y factores utilizados

\begin{tabular}{lccc}
\hline & $\alpha$ & FC & VME \\
\hline Activación & 0,89 & 0,811 & 0,580 \\
\hline Pensamiento creativo & 0,92 & 0,913 & 0,640 \\
\hline Diversión & 0,90 & 0,899 & 0,630 \\
\hline Total & 0,93 & 0,785 & 0,594 \\
\hline
\end{tabular}

Fuente: elaboración propia.

Una vez comprobada la fiabilidad de la escala, se lleva a cabo un análisis descriptivo preliminar sobre los datos. Para la suma total de cada una de las dimensiones (tablas 2, 3 y 4) se obtienen los siguientes valores: activación $(14,57 \pm 3,202)$, pensamiento creativo $(16,41 \pm 3,270)$ y diversión $(18,22 \pm$ $3,150)$. Pueden considerarse valores altos respecto al máximo posible (20).

También destacan, para la dimensión activación (tabla 2), por la parte superior, el ítem Act1 («Mientras jugaba me sentía activo»: 4,40 \pm 0,734) y, por la parte inferior, con menor puntuación obtenida, el ítem Act3 («Mientras jugaba me sentía frenético»: 3,05 $\pm 1,220$ ).

Tabla 2. Descriptivos y valoración de medias para la dimensión activación

\begin{tabular}{lcc}
\hline Activación & M & DT \\
\hline Act1: «Mientras jugaba me sentía activo» & 4,40 & 0,734 \\
\hline Act2: «Mientras jugaba me sentía nervioso» & 3,14 & 1,283 \\
\hline Act3: «Mientras jugaba me sentía frenético» & 3,05 & 1,220 \\
\hline Act4: «Mientras jugaba me sentía emocionado» & 3,97 & 0,907 \\
\hline Total & 14,57 & 3,202 \\
\hline
\end{tabular}

Fuente: elaboración propia.

De otro modo, en la dimensión pensamiento creativo (tabla 3) destacan los

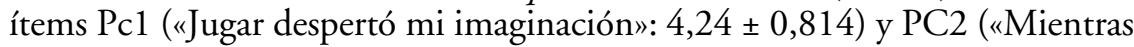
jugaba me sentí creativo»: $4,21 \pm 0,888$ y como valor mínimo destaca el ítem PC4 («Mientras jugaba me sentí innovador»: 3,94 $\pm 1,014$ ). 
Tabla 3. Descriptivos y valoración de medias para la dimensión pensamiento creativo

\begin{tabular}{lcc}
\hline Pensamiento creativo & M & DT \\
\hline PC1: «Jugar despertó mi imaginación» & 4,24 & 0,814 \\
\hline PC2: «Mientras jugaba me sentí creativo» & 4,21 & 0,888 \\
\hline PC3: «Mientras jugaba sentí que podía explorar cosas» & 4,01 & 0,970 \\
\hline PC4: «Mientras jugaba me sentí innovador» & 3,94 & 1,014 \\
\hline Total & 16,41 & 3,270 \\
\hline
\end{tabular}

Fuente: elaboración propia.

El mismo procedimiento se sigue para la dimensión diversión (tabla 4), donde destaca el ítem Div1 ("Jugar fue divertido»: 4,64 \pm 0,998) de forma positiva y, con el valor más bajo para la dimensión, el ítem Div4 («Mi experiencia con el juego fue placentera»: 4,02 $\pm 1,124$ ).

Tabla 4. Descriptivos y valoración de medias para la dimensión diversión

\begin{tabular}{lcc}
\hline Diversión & M & DT \\
\hline Div1: «Jugar fue divertido» & 4,64 & 0,998 \\
\hline Div2: «Me gustó jugar» & 4,56 & 1,388 \\
\hline Div3: «Disfruté mucho jugando» & 4,15 & 0,690 \\
Div4: «Mi experiencia con el juego fue placentera» & 4,02 & 1,124 \\
Total & 18,22 & 3,150 \\
\hline
\end{tabular}

Fuente: elaboración propia.

En general, aunque haya diferencia entre los valores superiores e inferiores, todos pueden considerarse muy buenos dentro de cada una de las dimensiones analizadas, ya que el valor máximo posible a conseguir en cada una era de $5 \mathrm{y}$ el valor mínimo, de 1 .

Siguiendo el análisis de los datos propuestos, se lleva a cabo la comparación de los resultados obtenidos en función del género de los participantes (tabla 5). No se encuentran diferencias significativas para la dimensión activación $(U=5.942,500, p=0,628)$, el pensamiento creativo $(U=5.639,000$, $p=0,858)$ o la diversión $(U=5.139,000, p=0,815)$.

Aunque sí cabe destacar que las puntuaciones obtenidas por las mujeres en el factor pensamiento creativo $(\mathrm{RP}=113,01)$ son superiores a las de los hombres $(\mathrm{RP}=111,43)$, al contrario de lo que sucede con la dimensión activación, en la que los hombres $(\mathrm{RP}=113,56)$ puntúan de forma superior a las mujeres $(\mathrm{RP}=109,22)$. 
Tabla 5. Prueba U de Mann Whitney para las dimensiones en función del género

\begin{tabular}{llcccc}
\hline & & $\mathbf{R P}^{*}$ & $\boldsymbol{U}$ & $\mathbf{Z}$ & $\boldsymbol{p}$ \\
\hline \multirow{2}{*}{ Activación } & Mujeres & 109,22 & \multirow{2}{*}{$5.942,500$} & $-3,315$ & 0,628 \\
& Hombres & 113,56 & & & \\
\multirow{2}{*}{ Pensamiento creativo } & Mujeres & 113,01 & \multirow{2}{*}{$5.639,000$} & $-2,021$ & 0,858 \\
& Hombres & 111,43 & & & \\
\multirow{2}{*}{ Diversión } & Mujeres & 111,01 & \multirow{2}{*}{$5.139,000$} & $-1,021$ & 0,815 \\
& Hombres & 111,43 & & & \\
\hline
\end{tabular}

${ }^{*} \mathrm{RP}$ : rangos promedio.

Fuente: elaboración propia.

De la misma manera, los análisis en función del tipo de participante (tabla 6) arrojan diferencias significativas para la dimensión activación $(U=$ $7.185,500, p=0,041)$ y para pensamiento creativo $(U=7.498,000, p=0,006)$, pero sucede lo mismo en diversión $(U=7.285,000, p=0,010)$.

Tanto el tamaño del efecto para activación $(d=0,26)$ como para pensamiento creativo $(d=0,36)$ pueden considerarse pequeños, lo que muestra que, aunque las diferencias sean significativas, estas no son amplias. En este caso, para la dimensión diversión no es posible calcular este estadístico, al no encontrarse diferencias significativas.

Tabla 6. Prueba U de Mann Whitney para las dimensiones en función del tipo de participante

\begin{tabular}{llrcccc}
\hline & & $\mathbf{R P}$ & $\boldsymbol{U}$ & $\boldsymbol{Z}$ & $\boldsymbol{p}$ & $\boldsymbol{d}$ \\
\hline \multirow{2}{*}{ Activación } & Docentes & 120,44 & $7.185,500$ & $-2,045$ & 0,041 & 0,26 \\
& Estudiantes & 102,85 & & & & \\
\multirow{2}{*}{ Pensamiento creativo } & Docentes & 123,14 & $7.498,000$ & $-2,731$ & 0,006 & 0,36 \\
& Estudiantes & 99,93 & & & & \\
Diversión & Docentes & 111,12 & $7.285,000$ & $-2,131$ & 0,010 & - \\
& Estudiantes & 100,93 & & & & \\
\hline
\end{tabular}

Fuente: elaboración propia.

Además, se realiza un contraste de hipótesis con el grupo de alumnos (tabla 7), divididos en función de los estudios que están cursando, si son de grado o de posgrado. No se encuentran diferencias significativas para la dimensión activación $(U=5.786,500, p=0,774)$, pensamiento creativo $(U=$ 5.789,000, $p=0,767)$ ni diversión $(U=5.386,000, p=0,864)$. 
Tabla 7. Prueba $U$ de Mann Whitney para las dimensiones en función del tipo de estudios que cursan

\begin{tabular}{llcccc}
\hline & & RP & U & Z & p \\
\hline \multirow{2}{*}{ Activación } & Posgrado & 111,09 & & & \\
& Grado & 113,69 & $5.786,500$ & $-0,288$ & 0,774 \\
\multirow{2}{*}{ Pensamiento creativo } & Posgrado & 111,00 & & & \\
& Grado & 113,00 & $5.789,000$ & $-0,297$ & 0,767 \\
\multirow{2}{*}{ Diversión } & Posgrado & 111,09 & & & \\
& Grado & 113,69 & $5.386,000$ & $-0,368$ & 0,864 \\
\hline
\end{tabular}

Fuente: elaboración propia.

Por último, se ponen a prueba las posibles diferencias entre los participantes según su edad. Para que la muestra pueda ser repartida de una forma homogénea, establecemos el valor medio para crear dos grupos de edad concretos de 26 años. Siguiendo los resultados obtenidos (tabla 8), no se encuentran diferencias significativas en función de la dimensión activación $(U=5.626,500, p=0,524)$, pensamiento creativo $(U=4.789,000, p=0,367)$ ni diversión $(U=5.286,000, p=0,634)$.

Tabla 8. Prueba U de Mann Whitney para las dimensiones en función de la edad

\begin{tabular}{lccccc}
\hline & & $\mathbf{R P}$ & $\boldsymbol{U}$ & $\boldsymbol{Z}$ & $\boldsymbol{p}$ \\
\hline \multirow{2}{*}{ Activación } & $<26$ & 109,10 & $5.626,500$ & $-0,381$ & 0,524 \\
& $>26$ & 111,69 & & & \\
\multirow{2}{*}{ Pensamiento creativo } & $<26$ & 112,10 & & & \\
& $>26$ & 115,00 & $4.789,000$ & $-0,360$ & 0,367 \\
Diversión & $<26$ & 109,10 & & & \\
& $>26$ & 103,32 & $5.286,000$ & $-0,408$ & 0,634 \\
\hline
\end{tabular}

Fuente: elaboración propia.

\section{Discusión}

Las decisiones de los maestros y profesores son cruciales en los procesos de enseñanza-aprendizaje, ya que desempeñan un papel clave en la introducción de innovaciones pedagógicas en el aula, especialmente en lo que se refiere a las que están relacionadas con la tecnología (Ketelhut y Schifter, 2011), por lo que los docentes desempeñarán un papel clave en la adopción de aquella por parte de sus alumnos y sus compañeros (Bourgonjon et al., 2013).

Los resultados arrojan evidencias acerca de los efectos positivos de las experiencias educativas gamificadas, tanto para profesores como para estudiantes. 
Este hecho ha sido bien contrastado en la literatura científica (Aznar-Díaz et al., 2016), que habitualmente muestra los efectos positivos de dichas experiencias sobre la motivación o la diversión (Alsawaier, 2018; Sailer, Hense, Mayr y Mandl, 2017).

Por otro lado, la activación es uno de los aspectos clave de cualquier acción educativa (Cassidy, 2004). Uno de los objetivos principales de cualquier plataforma gamificada es el de aumentar el compromiso del participante (Garaigordobil y Berrueco, 2011). Los resultados obtenidos muestran que las experiencias en las que se han visto inmersos los participantes han producido una activación de los mismos, y este efecto es significativamente más pronunciado en los docentes.

Aunque la gamificación está ganando fama en los últimos años, faltan estudios que revelen sus cualidades para fomentar la creatividad (Kalinauskas, 2014; Romero-Rodríguez, Torres-Toukoumidis, y Aguaded, 2016). Se ha demostrado la influencia de pequeñas experiencias gamificadas que producen efectos positivos en los estudiantes, aunque, al igual que sucede con los resultados obtenidos, las comparaciones entre la mayoría de las variables analizadas no muestran diferencias significativas (Garaigordobil y Berrueco, 2011; ParraGonzález, López-Belmonte, Segura-Robles y Fuentes-Cabrera, 2020). Destacan en nuestro caso los valores significativamente más elevados obtenidos por los docentes.

De otro modo, y aunque hay estudios que han encontrado diferencias significativas en el desarrollo de experiencias en contextos educativos relacionadas con la utilización de videojuegos en cuanto al género del usuario (Bressler y Bodzin, 2013), siguiendo los resultados obtenidos en esta investigación, la variable género no parece influir de manera significativa en ello cuando analizamos experiencias puramente gamificadas (Martí-Parreño, SeguíMas y Seguí-Mas, 2016).

\section{Conclusiones}

El análisis de los resultados permite detectar los efectos positivos que produce la gamificación entre los profesores y los estudiantes en cuanto a los altos niveles de motivación conseguidos. Pero no solamente aumenta esta, sino que además los alumnos están más activos durante el proceso de aprendizaje. Al estar activos, son más protagonistas y construyen su aprendizaje, con lo que el grado de compromiso se acrecienta. Los niveles de activación de los docentes también han sido bastante elevados, con lo que se desprende que a través de la gamificación aumenta tanto la activación de los alumnos como la de los profesores. En tercer lugar, la creatividad también se ha visto beneficiada, ya que muestra unos grados elevados. Son los docentes los que presentan mayores niveles de creatividad en relación con las experiencias gamificadas.

Defendemos aquí el uso de la gamificación como metodología educativa, ya que, como demuestra nuestro estudio, se obtienen diferentes beneficios muy provechosos, por ejemplo: presentar altos niveles de motivación en alum- 
nos y docentes, así como de activación y fomento de la creatividad. Esta metodología permite trabajar estas variables y obtener unos niveles de desarrollo de las mismas, que serían difícilmente alcanzables con metodologías tradicionales.

\section{Futuras líneas de investigación}

Como futuras líneas de investigación nos planteamos, por un lado, especificar qué tipos de experiencias gamificadas se desarrollan en torno a las distintas categorías, como pueden ser el uso de juegos de escape, búsquedas del tesoro, etc., y, por otro, comparar la diferencia de las variables analizadas en este estudio entre las enseñanzas en entornos virtuales y las enseñanzas presenciales, para ver si ese factor tiene algún efecto en el mundo académico.

\section{Referencias bibliográficas}

Alsawaier, R. (2018). The effect of gamification on motivation and engagement. International Journal of Information and Learning Technology, 35(1), 56-79. $<$ http://dx.doi.org/10.1108/IJILT-02-2017-0009>

Aznar-Díaz, I.; Raso-Sánchez, F.; Hinojo-Lucena, M.A. y Romero-Díaz de la GuARDia, J.J. (2016). Percepciones de los futuros docentes respecto al potencial de la ludificación y la inclusión de los videojuegos en los procesos de enseñanzaaprendizaje. Educar, 53(1), 11-28. $<$ http://dx.doi.org/10.5565/rev/educar.840>

Bourgonjon, J.; De Grove, F.; De Smet, C.; Van Looy, J.; Soetaert, R. y Valcke, M. (2013). Acceptance of game-based learning by secondary school teachers. Computers \& Education, 67, 21-35. $<$ http://dx.doi.org/10.1016/j.compedu.2013.02.010>

Bressler, D. y Bodzin, A. (2013). A mixed methods assessment of students' flow experiences during a mobile augmented reality science game. Journal of Computer Assisted Learning, 29(6), 505-517. $<$ http://dx.doi.org/10.1111/jcal.12008>

Brumels, K.A.; Blasius, T.; Cortright, T.; Oumedian, D. y Solberg, B. (2008). Comparison of efficacy between traditional and video game based balance programs. Clinical Kinesiology: Journal of the American Kinesiotherapy Association, 62(4), 26-32.

CAssidy, S. (2004). Learning Styles: An overview of theories, models, and measures. Educational Psychology, 24(4), 419-444. $<$ http://dx.doi.org/10.1080/0144341042000228834>

CoHen, J. (1987). Statistical power analysis for the behavioral sciences. Hillsdale: Erlbaum.

Corder, G. y Foreman, D. (2009). Nonparametric statistics for non-statisticians. Hoboken: Wiley.

Davidson, A. y Desjardins, F. (2011). Vers l'identification d'une relation entre les representations de la pédagogie et de l'usage des TIC chez des formateurs d'enseignants. Canadian Journal of Education / Revue canadienne de l'éducation, 34(3), 47-67.

Deterding, S.; Dixon, D.; Khaled, R. y Nacke, L. (2011). From Game Design Elements to Gamefulness: Defining "Gamification». Proceedings of the 15th Inter- 
national Academic MindTrek Conference on Envisioning Future Media Environments - MindTrek '11, 9-15. <http://dx.doi.org/10.1145/2181037.2181040>

Dunn, T.; Baguley, T. y Brunsden, V. (2013). From alpha to omega: A practical solution to the pervasive problem of internal consistency estimation. British Journal of Psychology, 105(3), 399-412. <http://dx.doi.org/10.1111/bjop.12046>

Eppmann, R.; BeкK, M. y Klein, K. (2018). Gameful Experience in Gamification: Construction and Validation of a Gameful Experience Scale [GAMEX]. Journal of Interactive Marketing, 43, 98-115. <http://dx.doi.org/10.1016/j.intmar.2018.03.002>

ERENLI, K. (2013). The impact of gamification: Recommending education scenarios. International Journal of Emerging Technologies in Learning, 8(1), 15-21. $<$ http://dx.doi.org/10.3991/ijet.v8iS1.2320>

FÁvero, L.P. y Belfiore, P. (2019). «Univariate Descriptive Statistics». En Data Science for Business and Decision Making (pp. 21-91). Amsterdam: Elsevier. <http://dx.doi.org/10.1016/B978-0-12-811216-8.00003-3>

Garaigordobil, M. y Berrueco, L. (2011). Effects of a Play Program on Creative Thinking of Preschool Children. The Spanish Journal of Psychology, 14(2), 608-618. $<$ http://dx.doi.org/10.5209/rev_SJOP.2011.v14.n2.9>

Gómez-García, S.; Planells de la Maza, A.J. y Chicharro-Merayo, M. (2016). ¿Los alumnos quieren aprender con videojuegos?: Lo que opinan sus usuarios del potencial educativo de este medio. Educar, 53(1), 49. $<$ http://dx.doi.org/10.5565/rev/educar.848>

Hair, J. y Gómez-Suárez, M. (2010). Análisis multivariante. Madrid: Prentice-Hall. Hassinger-Das, B.; Toub, T.S.; Zosh, J.M.; Michnick, J.; Golinkoff, R. y HirshPASEK, K. (2017). More than just fun: A place for games in playful learning / Más que diversión: El lugar de los juegos reglados en el aprendizaje lúdico. Infancia y Aprendizaje, 40(2), 191-218. <http://dx.doi.org/10.1080/02103702.2017.1292684>

HuizingA, J. (1955). Homo ludens: A study of the play element in culture. Boston: Beacon. Kalinauskas, M. (2014). Gamification in Fostering Creativity. Social Technologies, 4(1), 62-75. $<$ http://dx.doi.org/10.13165/ST-14-4-1-05>

Kapp, K.M. (2012). The Gamification of Learning and Instruction. San Francisco: John Wiley.

Ketelhut, D. y Schifter, C. (2011). Teachers and game-based learning: Improving understanding of how to increase efficacy of adoption. Computers \& Education, 56(2), 539-546. $<$ https://doi.org/10.1016/j.compedu.2010.10.002>

Kothari, C. (2004). Research methodology. Nueva Delhi: New Age International.

Lazzari, M. (2014). Combinación de Aprendizaje Cooperativo e Individual en una Asignatura de Química de Materiales. Formación Universitaria, 7(4), 39-46. <http://dx.doi.org/10.4067/S0718-50062014000400005>

Lee, J.J. y Hammer, J. (2011). Gamification in Education: What, How, Why Bother. Academic Exchange Quaterly, 15(2).

Martí-Parreño, J.; Seguí-Mas, D. y Seguí-Mas, E. (2016). Teachers' Attitude towards and Actual Use of Gamification. Procedia - Social and Behavioral Sciences, 228, 682-688. $<$ http://dx.doi.org/10.1016/j.sbspro.2016.07.104> 
Martínez, P.; Pérez, J. y Martínez, M. (2016). Las TICS y el entorno virtual para la tutoría universitaria. Educación XXI: Revista de la Facultad de Educación, 19(1), 287-310. <http://dx.doi.org/10.5944/educXX1.13942>

Martins dos Santos, G. y Mourâo, R.G. (2017). ICT in Education: Personal learning environments in perspectives and practices of young people. Educação $e$ Pesquisa AHEAD, 21, 1-20. <http://dx.doi.org/10.1590/s1678-4634201702153673>

MÉndeZ, R. (2008). Los jóvenes universitarios y su (des)orientación ante los nuevos retos que plantea el EEES. Educatio Siglo XXI, 26, 197-224.

MÉndeZ, R. y TRILlo, F. (2008). El papel de la información universitaria en el proceso de formación de actitudes de los estudiantes hacia la Universidad. Revista de Educación, 353, 329-360.

Mirate, A. y García, F. (2014). Rendimiento académico y TIC: Una experiencia con webs didácticas en la Universidad de Murcia. Pixel-Bit: Revista de Medios y Educación, 44, 69-183.

Muñoz-Carril, P.; Fuentes, E. y González-Sanmamed, M. (2012). Necesidades formativas del profesorado universitario en infografía y multimedia. Revista de Investigación Educativa, 30(2), 303-321. <http://dx.doi.org/10.6018/rie.30.2.118971>

Norman, G. (2010). Likert scales, levels of measurement and the "laws» of statistics. Advances in Health Sciences Education, 15(5), 625-632. <http://dx.doi.org/10.1007/s10459-010-9222-y>

Parra-González, M.E.; López-Belmonte, J.; Segura-Robles, A. y Fuentes-CabreRA, A. (2020). Active and Emerging Methodologies for Ubiquitous Education: Potentials of Flipped Learning and Gamification. Sustainability, 12(2), 1-11. <http://dx.doi.org/10.3390/su12020602>

Parra-González, M.E. y Segura-Robles, A. (2019). Producción científica sobre gamificación en educación: Un análisis cienciométrico. Revista de Educación, 5(386), 113-131. <http://dx.doi.org/10.4438/1988-592X-RE-2019-386-429>

Pérez-López, I. y Rivera García, E. (2017). Formar docentes, formar personas: Análisis de los aprendizajes logrados por estudiantes universitarios desde una experiencia de gamificación. Signo y Pensamiento, 36(70), 112-129. <https://doi.org/10.11144/Javeriana.syp36-70.fdfp>

Pintrich, P.R. y Schunk, D.H. (2006). Motivación en contextos educativos: Teoria, investigación y aplicaciones. 2a ed. Madrid: Pearson Prentice Hall.

Piñeiro-Otero, T. y Costa-SÁnchez, C. (2015). ARG (juegos de realidad alternativa): Contribuciones, limitaciones y potencialidades para la docencia universitaria. Comunicar, 44(22), 141-148. <http://dx.doi.org/10.3916/C44-2015-15>

Rayhan, R.U.; Zheng, Y.; Uddin, E.; Timbol, C.; Adewuyi, O. y Baraniuk, J.N. (2013). Administer and collect medical questionnaires with Google documents: A simple, safe, and free system. Applied Medical Informatics, 33(3), 12-21.

Ricoy, M. y Couto, M. (2014). Best practices with ICT and the value attributed by the students newly integrated in university. Educação e Pesquisa, 40(4), 897-912.

Rodríguez, A.B.; Ramírez Leonardo, J. y Fernández, W. (2017). Metodologías Activas para Alcanzar el Comprender. Formación Universitaria, 10(1), 79-88. <http://dx.doi.org/10.4067/S0718-50062017000100009> 
Romero-Rodríguez, L.-M.; Torres-Toukoumidis, Á. y AguAded, I. (20I6). Ludificación y educación para la ciudadanía. Revisión de las experiencias significativas. Educar, 53(1), 109-128. https://doi.org/10.5565/rev/educar.846

Rubilar, P.; Alveal, F. y Fuentes, A. (2017). Evaluation of digital and pedagogical literacy in ICT based on the opinions of Initial Teacher Education students. Educação e Pesquisa, 43(1), 127-143. <http://dx.doi.org/10.1590/s1517-9702201701154907>

Sailer, M.; Hense, J.; MaYr, S. y Mandl, H. (2017). How gamification motivates: An experimental study of the effects of specific game design elements on psychological need satisfaction. Computers in Human Behavior, 69, 371-380. $<$ http://dx.doi.org/10.1016/j.chb.2016.12.033>

Segura-Robles, A. y Parra-González, M.E. (2019). Metodologías activas: El uso del Escape Room en el aula. En A. Baena-Extremera y P. Ruiz-Montero (eds.). Metodologias Activas en Ciencias de la Educación, I (pp. 285-298). Barcelona: Wanceulen.

Shneiderman, B. (2004). Designing for Fun: How Can We Design User Interfaces to Be More Fun? Interactions, 11(5), 48-50.

Sullivan, G. y Feinn, R. (2012). Using Effect Size-or Why the PValue Is Not Enough. Journal of Graduate Medical Education, 4(3), 279-282. <http://dx.doi.org/10.4300/JGME-D-12-00156.1>

Teixes, F. (2015). Gamificación: Fundamentos y aplicaciones. Barcelona: UOC.

Vickers, A. (2005). Parametric versus non-parametric statistics in the analysis of randomized trials with non-normally distributed data. BMC Medical Research Methodology, 5(1), 35-40. $<$ http://dx.doi.org/10.1186/1471-2288-5-35>

Von Ahn, L. y Dabbish, L. (2008). Designing games with a purpose. Communications of the $A C M, 51(8), 57$. <http://dx.doi.org/10.1145/1378704.1378719>

WANG, A. (2015). The wear out effect of a game-based student response system. Computers \& Education, 82, 217-227. $<$ http://dx.doi.org/10.1016/j.compedu.2014.11.004>

Werbach, K. (2014). (Re)Defining Gamification: A Process Approach. En A. SpaGnolli, L. Chittaro y L. Gamberini (eds.). Persuasive Technology (pp. 266-272). Proceedings of 9th International Conference, PERSUASIVE 2014, Padua, Italy, May 21-23, 2014. <http://dx.doi.org/10.1007/978-3-319-07127-5_23>

ZaINUDDIN, Z. (2018). Students' learning performance and perceived motivation in gamified flipped-class instruction. Computers \& Education, 126, 75-88. <http://dx.doi.org/10.1016/j.compedu.2018.07.003>

Zumbo, B.; Gadermann, A. y Zeisser, C. (2007). Ordinal Versions of Coefficients Alpha and Theta for Likert Rating Scales. Journal of Modern Applied Statistical Methods, 6(1), 21-29.

$<$ http://dx.doi.org/10.22237/jmasm/1177992180> 